\title{
Turkish pediatric atypical hemolytic uremic syndrome registry: initial analysis of 146 patients
}

\author{
Nesrin Besbas', Bora Gulhan', Oguz Soylemezoglu², Z. Birsin Ozcakar ${ }^{3}$ Emine Korkmaz', Mutlu Hayran \\ and Fatih Ozaltin ${ }^{1 *}$ (1)
}

\begin{abstract}
Background: Atypical hemolytic uremic syndrome (aHUS) is a devastating disease with significant morbidity and mortality. Its genetic heterogeneity impacts its clinical presentation, progress, and outcome, and there is no consensus on its clinical management.

Methods: To identify the characteristics of aHUS in Turkish children, an industry-independent registry was established for data collection that includes both retrospective and prospective patients.

Results: In total, 146 patients (62 boys, 84 girls) were enrolled; 53 patients (36.3\%) were less than 2 years old at initial presentation. Among the 42 patients (37.1\%) whose mutation screening was complete for CFH, CFI, MCP, CFB, C3, DGKE, and CHFR5 genes, underlying genetic abnormalities were uncovered in 34 patients (80.9\%). Sixty-one patients (41.7\%) had extrarenal involvement. During the acute stage, 33 patients (22.6\%) received plasma therapy alone, among them 17 patients (51.5\%) required dialysis, and 4 patients (12.1\%) were still on dialysis at the time of discharge. In total, 103 patients (70.5\%) received eculizumab therapy, 16 of whom (15.5\%) received eculizumab as a first-line therapy. Plasma therapy was administered to $84.5 \%$ of the patients prior to eculizumab. In this group, renal replacement therapy was administered to 80 patients (77.7\%) during the acute period. A total of 3 patients died during the acute stage. A total of 101 patients (77.7\%) had a glomerular filtration rate $>90 \mathrm{~mL} / \mathrm{min} / 1.73 \mathrm{~m}^{2}$ at the 2-year follow-up.
\end{abstract}

Conclusions: The Turkish aHUS registry will increase our knowledge of patients with aHUS who have different genetic backgrounds and will enable evaluation of the different treatment options and outcomes.

Keywords: Atypical hemolytic uremic syndrome, Turkish registry, Treatment, Outcome, Prognosis

\section{Background}

Atypical hemolytic uremic syndrome (aHUS) is a lifethreatening systemic disease associated with the dysregulation of the complement system [1]. It has been reported that mortality was higher in children when compared to adults whereas progression to end stage renal disease after the first episodes was higher in adults [2]. Mutations in the genes encoding complement regulatory proteins (i.e. $C F H, C F I, M C P$ (CD46), or $C F H$-CFHR genomic rearrangements) and components

\footnotetext{
* Correspondence: fozaltin@hacettepe.edu.tr

${ }^{1}$ Department of Pediatric Nephrology, Nephrogenetics Laboratory, Hacettepe

University Faculty of Medicine, Sihhiye 06100, Ankara, Turkey

Full list of author information is available at the end of the article
}

of the alternative pathway C3 convertase (i.e. C3 and $C F B$ ) or anti-complement factor $\mathrm{H}$ autoantibodies are identified in $60-70 \%$ of patients with aHUS [2-7]. Recent studies have also identified mutations in diacylglycerol kinase- $\varepsilon$ (DGKE), thrombomodulin (THBD, CD141) in thrombotic microangiopathies (TMAs) including aHUS [8-13]. For years, plasma therapy was the mainstay of treatment for aHUS [11, 12]. Eculizumab, a monoclonal antibody that blocks the terminal part of the complement system, has revolutionized the prognosis of aHUS and is currently recommended as first-line therapy in children with aHUS, whenever possible [11]. Several retrospective and prospective studies involving both pediatric and adult patients have demonstrated the 
efficacy and safety of eculizumab [11, 14, 15]. However, exact duration of therapy and dose intervals are still a matter of debate.

Owing to the low incidence of aHUS, large patient registries are required to adequately address such questions and to evaluate the natural history and progression of the disease, and the prognosis of patients from different ethnic backgrounds. One of the largest aHUS registries, the global aHUS registry, was established in 2012 with industry support; it includes multiple participating centers from 16 countries, not including Turkey. This registry prospectively collects information on patient demographics, disease characteristics, and treatment modalities [16]. In addition, there are a few registries of patients from different ethnic backgrounds [2, 17, 18]. However, investigators are aware that underlying genetic abnormalities of patients from different ethnic backgrounds differ and that the characteristics of aHUS may vary among different patient populations. In Turkey, an industry-independent aHUS registry was established to collect data on pediatric patients with aHUS who were treated in the 26 pediatric nephrology centers. This registry not only collects information on the demographic, clinical, laboratory, and genetic features of Turkish pediatric patients with aHUS, but also affords researchers a unique opportunity to evaluate the treatment strategies used at the different centers and to assess their impact on the long-term prognosis of the patients. This study aimed to introduce the registry and to provide a general perspective about its initial findings. Specific analyses addressing specific questions (i.e. genetics, long-term prognosis etc.) will be conducted in our subsequent studies.

\section{Methods}

\section{Definitions}

Pediatric aHUS was defined as a triad of Coombs-negative microangiopathic hemolytic anemia, thrombocytopenia, and acute renal failure in patients younger the age of 18 years. No adult patient, even if the first episode was observed during the childhood period, was included. Shiga toxin-producing E. coli (STEC) was investigated in a national centralized reference laboratory and STEC positive patients were excluded. Patients with depressed ADAMTS13 levels (i.e. $\leq 5 \%$ ), other specific infections, coexisting diseases or those with drug-related HUS were also excluded [11].

Hematologic remission was defined as platelet count $\geq 150,000 / \mathrm{mm}^{3}$ and lactate dehydrogenase levels $\leq$ upper limits of normal for $\geq 2$ consecutive measurements taken $\geq 4$ weeks apart, and cessation of hemolysis [11].

Renal remission was defined as estimated glomerular filtration rate $(\mathrm{eGFR})>90 \mathrm{~mL} / \mathrm{min} / 1.73 \mathrm{~m}^{2}$.

\section{Data collection}

Beginning year 2013, all retrospective and prospective pediatric patients with aHUS from 26 pediatric nephrology centers have been registered in the database at www.ahusnet.org, a web-based national registry system (NRS). A pediatric nephrology specialist from each participating center is responsible for entering patients into the registry. The objectives of the NRS are to assess the clinical and genetic characteristics, treatment modalities, associated extrarenal findings, and clinical outcomes of patients with aHUS during their initial hospital admission, and to evaluate their long-term prognosis according to disease management strategies.

Responsible specialists from each center registered both retrospective and prospective patients in the secure NRS and were able to access the website at any time to enter and edit data. During patient registration, the following data were entered into the system: demographics, medical and disease history, clinical characteristics at disease onset (i.e. physical examination findings and laboratory data), genetic results (if available), all therapies implemented during the acute stage (i.e. plasma therapy including plasma infusions and/or plasma exchange, eculizumab, rituximab, corticosteroid, antihypertensive, hemodialysis, peritoneal dialysis, and continuous renal replacement therapies), and the renal/hematologic status (i.e. proteinuria, hypertension, serum creatinine level, hemoglobin level, and platelet count) of the patient at discharge. The side effects related to any of the treatment medications were also recorded in the NRS. Follow-up data, including the hematologic and renal parameters of the patient, current therapies, and relapses (if any, since the last visit), were entered into the NRS every 3 months. All patients in the NRS were evaluated annually by the joint committee, which comprised pediatric nephrology experts. Any patient who did not meet the criteria for aHUS, as determined by the joint committee, was removed from the registry.

\section{Genetic analyses and ELISA}

Genetic testing was not a prerequisite for patient registration. However, if individual genetic data was available, their registration was also requested. DNA sample was available in 113 patients. Mutation analyses via Sanger sequencing of the coding regions of all of the following genes were performed in 42 patients (37.1\%) at the Nephrogenetics Laboratory of Hacettepe University: CFH, CFI, MCP, CFB, C3, DGKE, and CHFR5. Where applicable, in slico analyses, using Sorting Tolerant From Intolerant (SIFT) (http://sift.jcvi.org), Polymorphism Phenotyping v2 (PolyPhen2) (http://genetics.bwh.harvard.edu/pph2/index.shtml), Mutation taster (http:// www.mutationtaster.org), and Human Splicing Finder (http://www.umd.be/HSF3/index.html) softwares were 
applied for predicting likely effects of the variations. aHUS mutation database (http://www.fh-hus.org), The Human Gene Mutation Database Professional (http:// www.hgmd.cf.ac.uk/ac/index.php) and dbSNP database (https://www.ncbi.nlm.nih.gov/snp) were used to check whether identified variations had been reported previously. CFHR1-3 deletion in patients with anti-CFH autoantibodies was evaluated by multiplex ligationdependent probe amplification (MLPA) analyses. Anticomplement Factor $\mathrm{H}$ autoantibody was searched in 44 patients using the CFH IgG ELISA Kit (Abnova $\left.{ }^{\mathrm{Tm}}\right)$, according to the manufacturer's recommendations, with a detection limit of $0.6 \mathrm{AU} / \mathrm{mL}$. ADAMTS13 activity was detected using the ADAMTS-13 Activity Kit (Technozym ${ }^{\mathrm{Tw}}$ ) according to the manufacturer's recommendations, with a detection limit of $0.2 \%$ and an assay range of $0.3-105 \%$.

The study was approved by the ethics committee of Hacettepe University (FON10/03-22). Written informed consent was obtained from the parents of all the patients.

\section{Statistical analysis}

Descriptive statistical analysis methods were used to evaluate the demographics and clinical data. The mean, median, standard deviation, and interquartile range (IQR) were calculated for the numeric variables. Frequency tables were used to describe the categorical data. Mann Whitney $U$ test was used to compare 2 independent samples. Data were analyzed using SPSS v.21 (SPSS Inc., Chicago, IL, USA).

\section{Results}

\section{Patient characteristics}

As of March 30, 2016, 146 patients (62 boys, 84 girls) from 26 pediatric nephrology centers were enrolled in the NRS. The demographic and clinical characteristics of the patients are presented in Table 1 . A total of 69 patients $(47.6 \%)$ had diarrhea prior to their initial presentation, and 35 patients $(24.1 \%)$ had preceding upper respiratory tract infection. Notably, 53 of the 146 patients $(36.3 \%)$ were aged less than 2 years at the first presentation, and 29 of the patients $(19.8 \%)$ were aged less than 1 year. A total of 61 patients (41.7\%) showed extra-renal involvement; the most common site of extrarenal manifestation was the neurologic system $(n=41$; 28.1\%). Hemiparesis, loss of vision, unconsciousness, headache, hallucination, encephalopathy were the main symptoms for the neurological involvement. In 8 of these patients, another system was also involved. Brain imaging was performed in 27 patients, 15 of whom showed abnormal radiological findings. Cardiac complications were identified in 9 patients (6.1\%); 2 patients had hypertrophic cardiomyopathy, and the other cardiac
Table 1 Demographic and clinical characteristics of 146 patients with aHUS

\begin{tabular}{|c|c|}
\hline Male (\%) / Female (\%) & $\begin{array}{l}62(42.4) / 84 \\
(57.6)\end{array}$ \\
\hline \multicolumn{2}{|l|}{ Current age, years } \\
\hline Mean (SD) & $7.98(5.0)$ \\
\hline Median (IQR) & $6.53(3.8-11)$ \\
\hline \multicolumn{2}{|l|}{ Age at diagnosis, years } \\
\hline Mean (SD) & $4.82(4.4)$ \\
\hline Median (IQR) & $3.5(1.2-7.4)$ \\
\hline \multicolumn{2}{|l|}{ Age category (years) at the time of diagnosis, $\mathrm{n}(\%)$} \\
\hline$<2$ & $53(36.3)$ \\
\hline 2 to $<5$ & $40(27.4)$ \\
\hline 5 to $<12$ & $38(26)$ \\
\hline$\geq 12$ & $15(10.3)$ \\
\hline \multicolumn{2}{|l|}{ Duration of follow-up (years) } \\
\hline Mean (SD) & $2.9(2.5)$ \\
\hline Median (IQR) & $2.1(1.2-3.8)$ \\
\hline Consanguinity (\%) & $42(28.8)$ \\
\hline Family history of aHUS (\%) & $7(4.8)$ \\
\hline Anuria (\%) /oliguria at admission (\%) & $\begin{array}{l}41(28) / 67 \\
(45.8)\end{array}$ \\
\hline \multicolumn{2}{|l|}{ Duration of anuria at admission (days) } \\
\hline Mean (SD) & $6.3(4.5)$ \\
\hline Median (IQR) & $5(2-10)$ \\
\hline \multicolumn{2}{|l|}{ Duration of oliguria at admission (days) } \\
\hline Mean (SD) & $6(6.8)$ \\
\hline Median (IQR) & $3(2-7)$ \\
\hline Extrarenal involvement, n (\%) & $61(41.7)$ \\
\hline Central nervous system & $41(28.1)$ \\
\hline Gastrointestinal system & $16(10.9)$ \\
\hline Cardiac & $9(6.1)$ \\
\hline Respiratory system & $10(6.8)$ \\
\hline $\begin{array}{l}\text { Patients with GFR }<90 \mathrm{~mL} / \mathrm{min} / 1.73 \mathrm{~m}^{2} \text { at admission, } \\
\mathrm{n}(\%)\end{array}$ & $139(95.2)$ \\
\hline Hypocomplementemia (\%) ${ }^{a}$ & $66(48.5)$ \\
\hline Renal biopsy at admission (\%) & $44(30.1 \%)$ \\
\hline
\end{tabular}

SD: standard deviation; IQR: interquartile range; aHUS: atypical hemolytic

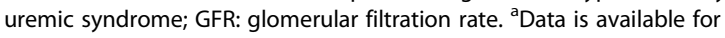
136 patients

manifestations included dilated cardiomyopathy, intracardiac thrombus, elevated creatine kinase (CK)-MB levels, mitral and tricuspid insufficiency, aortic valve insufficiency, left ventricular hypertrophy, and tachycardia. Involvement of the gastrointestinal system was observed in 16 children (10.9\%) and pancreatitis, elevated liver enzymes, invagination, ischemic hepatitis, cholelithiasis, vomiting were the main symptoms. GI bleeding and pancreatitis were the most common 
findings, which were observed in 4 and 3 patients, respectively. Respiratory involvement was observed in 10 patients $(6.8 \%)$, while 16 patients $(10.9 \%)$ showed multisystem involvement. Kidney biopsies were performed in 44 patients. The biopsies were performed to confirm the diagnosis (in 29 patients), before initiation of eculizumab therapy (in 7 patients), to assess patients with nephroticrange proteinuria (in 6 patients), and to evaluate patients who exhibited a poor response to plasma therapy or eculizumab (in 2 patients).

\section{Genetics}

Number of mutation analyses performed in individual genes are as follows in 113 patients whose DNA sample was available: $C F H(n=64,56.6 \%), C F 1(n=69,61.0 \%)$, $M C P(n=49,43.3 \%), C F B(n=62,54.8 \%), C 3 \quad(n=35$, $30.9 \%), D G K E(n=67,59.2 \%)$. Mutation screening was complete for all of the corresponding genes in 42 patients (37.1\%), and underlying genetic abnormalities were uncovered in 34 of these patients $(80.9 \%)$. These include isolated abnormality in $M C P(n=7,16.3 \%)$, in DGKE $(n=6,8.9 \%)$, in C3 $(n=4,11.4 \%)$, in CFH $(n=5$, $7.8 \%)$, in $C F B(n=1,1.6 \%)$, and in $C F I(n=1,1.4 \%)$. In 4 patients, combined genetic abnormalities were detected: CFH/CFB mutation ( $n=1), C F B$ mutation/CFHR1-3 deletion ( $\mathrm{n}=1), C F B$ mutation/CFHR1-3 deletion/anti-CFH autoantibody $(n=1)$, and CFHR5 mutation/CFHR1-3 deletion/anti-CFH autoantibody $(n=1)$. In addition, 1 patient was diagnosed with cobalamin deficiency. The anti$\mathrm{CFH}$ autoantibody was detected in 5 out of 44 patients who had been studied for anti-CFH autoantibody (11.3\%). All of these 5 patients had homozygous CFHR13 deletion. To our best of knowledge, 17 novel variations have been identified in this study (Table 2).

\section{Treatment}

Remission was achieved with supportive therapy in 5 patients and by cobalamin administration, in 1 patient. Four patients were diagnosed with ESKD at the time of admission and, therefore, did not receive specific therapy. During the acute stage, 33 patients (22.6\%; M/F 13/ 20) were treated with plasma therapy alone (i.e. plasma infusion and/or plasma exchange). Of these, 15 patients received plasma infusions, 14 patients underwent plasma exchanges, and 4 patients received both therapies. The median age at diagnosis in this group of patients was 3.9 (IQR $1.8-8.3)$ years. In total, 17 patients $(51.5 \%)$ required dialysis; 2 patients (6\%) died during the acute stage. At the time of discharge, 4 patients $(12.1 \%)$ were on dialysis, 26 patients (78.7\%) did not require dialysis, and data on 1 patient were not available. The median follow-up time for this group of patients was 1.7 (IQR 1.1-5.3) years. None of the patients required plasma therapy after discharge. At the last follow-up, 1 patient was still on dialysis and 1 patient had undergone renal transplantation; no other renal replacement therapies were recorded for the remaining patients. At the last follow-up visit, the GFR of 23 of the patients (69.6\%) was $>90 \mathrm{~mL} / \mathrm{min} / 1.73 \mathrm{~m}^{2}$.

In this registry population, 103 patients (70.5\%) received eculizumab therapy ( 45 males, 58 females). The median age at diagnosis in this group of patients was 3.4 (IQR 1.2-7) years, and the median follow-up duration was 2.05 (IQR 1.1-3.2) years. Eighty patients (77.7\%) underwent renal replacement therapy during the acute period. Plasma therapy was administered to $84.5 \%$ of the patients prior to the initiation of eculizumab, and $16 \mathrm{pa}-$ tients $(15.5 \%)$ received eculizumab as a first-line therapy. Complete hematologic and renal remission was achieved in $82.2 \%$ and $76.3 \%$ of the patients, respectively. Median follow up duration was 2.05 years (IQR 1.1-3.2 years) for this group. During the follow-up period, eculizumab treatment was discontinued in 52 patients (51.5\%) for various reasons (drug unavailability, genetic background of the patient, choice of the patient's family, or the clinician's decision). Five patients (4.8\%) with severe renal disease did not respond to therapy and progressed to ESKD. The initial median eGFR of these patients was significantly low when compared with the eGFRs of the rest of the patients $\left(7.6 \mathrm{~mL} / \mathrm{min} / 1.73 \mathrm{~m}^{2}\right.$ [range, 5.9 $18.3 \mathrm{~mL} / \mathrm{min} / 1.73 \mathrm{~m}^{2}$ ] vs. $18.6 \mathrm{~mL} / \mathrm{min} / 1.73 \mathrm{~m}^{2}$ [range, $\left.\left.0.3-166 \mathrm{~mL} / \mathrm{min} / 1.73 \mathrm{~m}^{2}\right] ; P=0.019\right)$. At the last followup visit, the GFR of 68 of the patients (66\%) was $>90 \mathrm{~mL} / \mathrm{min} / 1.73 \mathrm{~m}^{2}$. Eculizumab-related side effects were reported in 2 patients: an allergic reaction in 1 patient, and convulsions and sepsis after eculizumab administration in 1 patient. No patient relapsed while receiving eculizumab therapy.

\section{Follow-up}

A total of 3 patients died during the acute stage. The causes of death included sepsis $(n=1)$, intractable seizures $(n=1)$, and multisystem involvement $(n=1)$. Three patients died during the follow-up period. A total of 10 patients were lost to follow-up, and outcome data were available for the remaining 130 patients (56 males, 74 females). At their last follow-up visit, 13 patients had undergone treatment for ESKD (hemodialysis $[n=5]$, peritoneal dialysis $[n=3]$, and renal transplantation $[n=$ 5]). More than $75 \%$ of the patients had a GFR $>90 \mathrm{~mL} /$ $\mathrm{min} / 1.73 \mathrm{~m}^{2}$ (Table 3).

\section{Discussion}

Here, we introduce one of the largest pediatric aHUS registries in the world. However, it is very well known that registries have its own limitations. In aHUS, many factors such as STEC, genetics, anti-factor $\mathrm{H}$ antibodies and follow-up data of the patients have to be taken into 


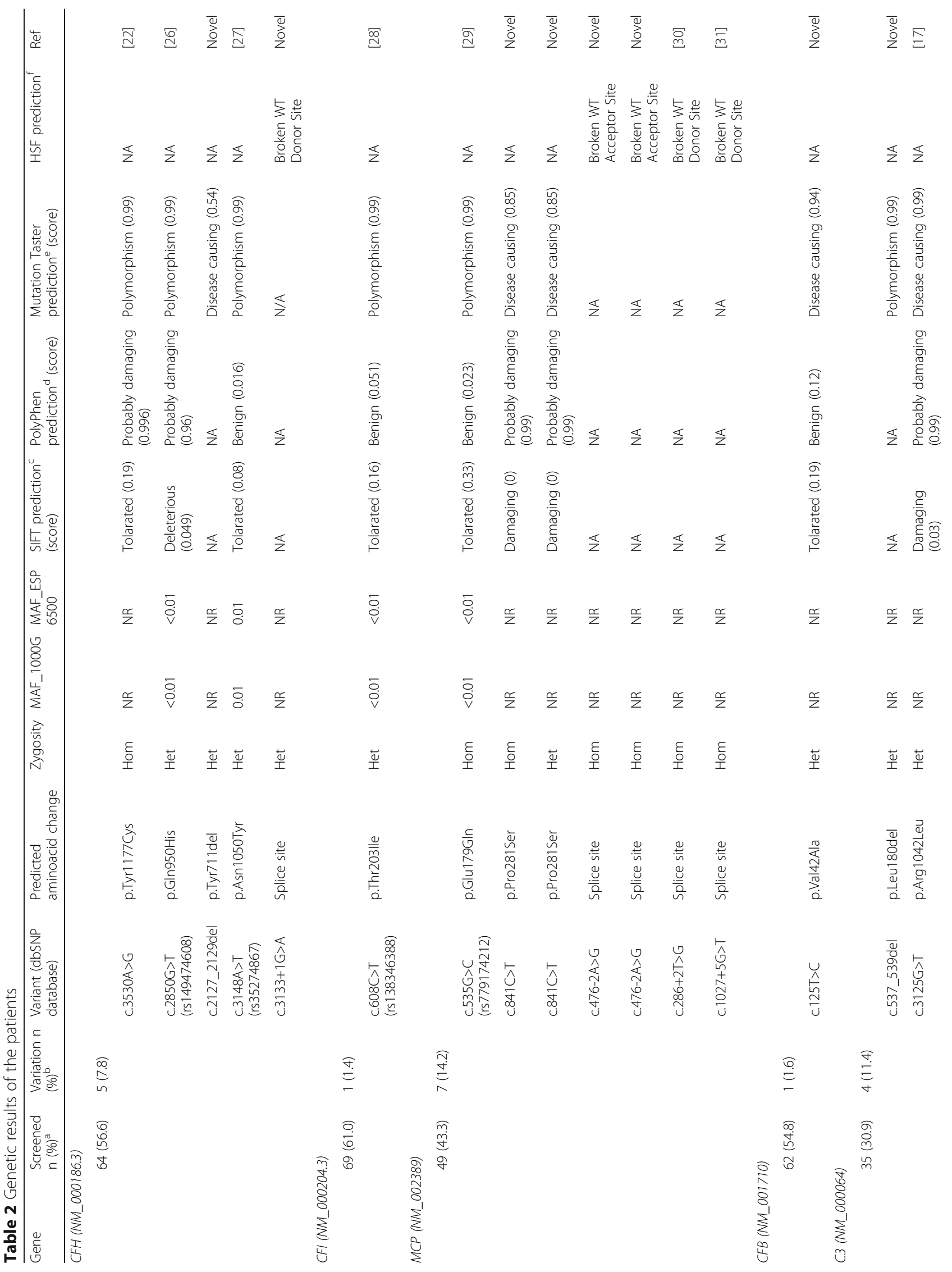




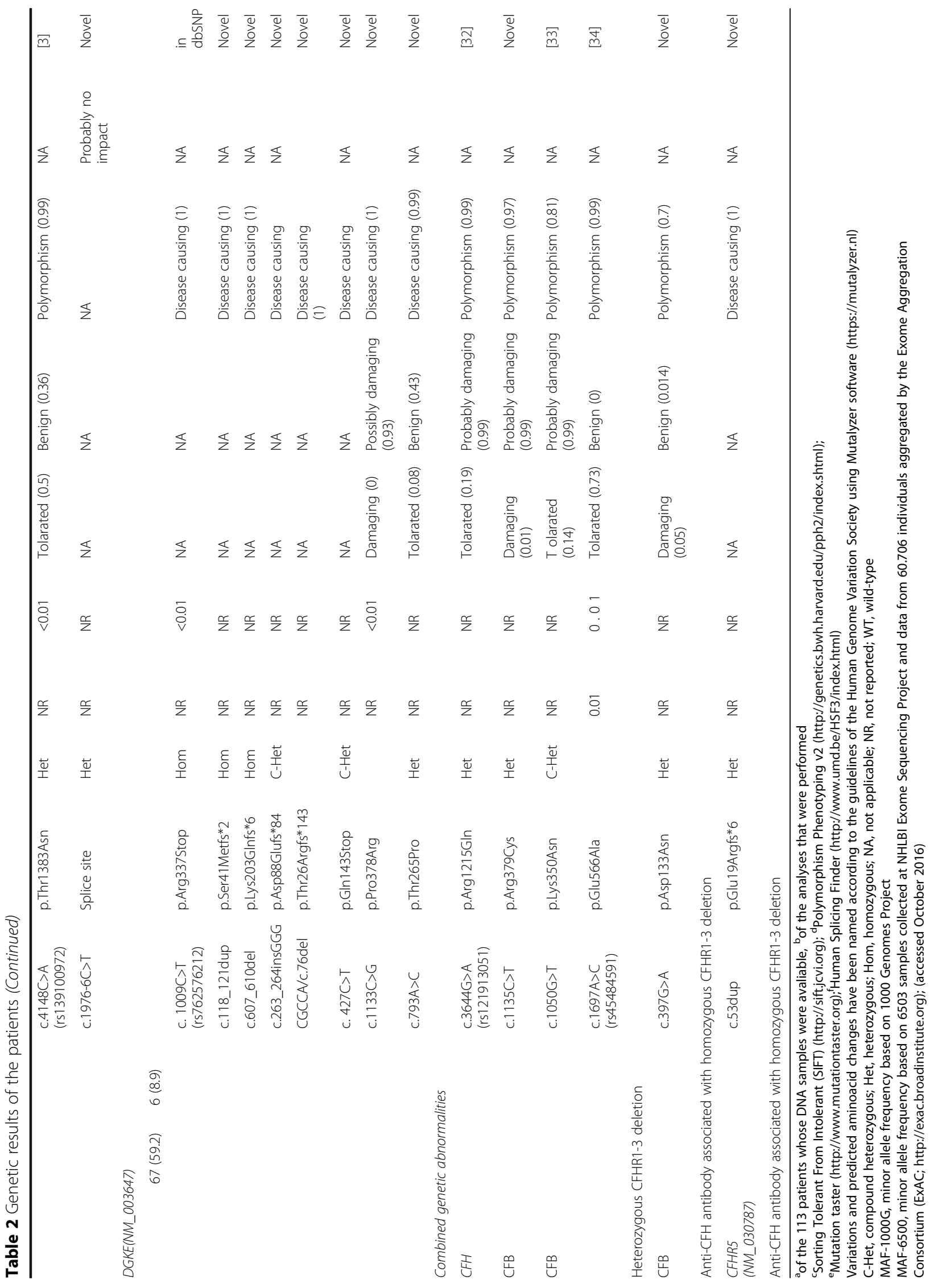


Table 3 Follow-up characteristics of patients $(n=130)$

\begin{tabular}{ll}
\hline Age at diagnosis, years & \\
Mean (SD) & $4.8(4.4)$ \\
Median (IQR) & $3.5(1.2-7.1)$ \\
Duration of follow-up (years) & \\
$\quad$ Mean (SD) & $2.8(2.3)$ \\
Median (IQR) & $2.1(1.2-3.7)$ \\
Renal replacement therapy ${ }^{a}, \mathrm{n}(\%)$ & $13(10)$ \\
GFR (mL/min/1.73 $\mathrm{m}^{2}$ ) at the last visit, $\mathrm{n}(\%)$ & \\
$>90$ & $101(77.7)$ \\
$60-89$ & $14(10.8)$ \\
$30-59$ & $6(4.6)$ \\
$15-29$ & $3(2.3)$ \\
$0-14$ & $6(4.6)$ \\
\hline
\end{tabular}

$S D$ standard deviation, IQR interquartile range, GFR glomerular filtration rate ancludes hemodialysis, peritoneal dialysis, and renal transplantation

consideration to draw a certain conclusion. In this study, we performed longitudinal follow-ups of pediatric patients with aHUS to evaluate their clinical and genetic characteristics in order to better understand the diversity of this rare disease in the Turkish population. Owing to the low incidence of aHUS, majority of the previous series on aHUS have included both adult and pediatric patients [2, 16-18] However, pediatric aHUS cases have different disease characteristics than those of adult cases. A study by Fremeaux-Bacchi et al. consisted of 214 patients, 89 of whom were pediatric patients. In this study, the characteristics of adult and pediatric patients were compared [2]. Another analysis of aHUS was from the global aHUS registry, which was supported by an industry sponsor. In that study, the baseline demographic and clinical characteristics of 516 patients with aHUS from 195 clinical centers in 16 countries were described. In that series, 201 patients were in the pediatric age group [16]. When compared with other established series, the Turkish aHUS registry has several distinctive features, namely its homogenous nature (i.e. it involves only Turkish patients with aHUS); availability of detailed data on patient demographics, initial clinical presentation, and the different treatments employed at the clinical centers; and availability of long-term follow-up and outcome data.

In our study, there were more female patients than male patients. While the mean patient age at diagnosis was comparable to the mean age of the population of the global aHUS registry, it was higher than the mean age of the population in the French registry (4.8 years vs. 1.5 years). Patients aged younger than 2 years accounted for $36.3 \%$ of the population in our study, which was significantly lower than the corresponding percentages reported in the global and French registries (43.9\% and
$56 \%$, respectively) $[2,16]$. A total of 28 patients (19.1\%) in our study were aged under 1 year; the corresponding data are not available in the other registries. In our study, patients aged older than 12 years constituted $10.3 \%$ of all patients, whereas this figure was $6.5 \%$ in the global registry. All these discrepancies could be attributed to the different genetic abnormalities and/or environmental factors that the patients in the different populations were exposed to. It is well known that some genetic abnormalities are associated with early-onset aHUS whereas others can cause adolescent- or even adult-onset aHUS. One of the most striking findings in our study was the rather low percentage of patients with a family history of aHUS when compared to the global registry $(4.8 \%$ vs $20.4 \%)$ despite a high rate of consanguinity in the Turkish population [16]. This was an unexpected finding and may be due to the fact that family history was not examined thoroughly by the reporting clinicians in our study. Alternatively, since the diagnosis of aHUS requires specific knowledge and expertise, aHUS among family members of the Turkish registry patients may not have been recognized if they did not undergo a specialist evaluation.

Extra-renal involvement in aHUS can significantly impact patient prognosis. Neurologic involvement is the most frequent complication, which can occur in 8-30\% of the patients [10]. In our study, neurologic involvement was observed in $28.1 \%$ of the patients, which is consistent with the literature. The involvement of other organ systems is reported to be much lower than that of the central nervous system. Johnson et al. reported that the most common gastrointestinal manifestations were pancreatitis/pancreatic insufficiency and elevated transaminases, and they observed respiratory and cardiac involvement (i.e. heart failure/cardiomyopathy and pericardial effusion) in $21 \%$ and $5.6 \%$ of pediatric patients with aHUS, respectively [12]. In the current study, we observed that pancreatitis was one of the most common gastrointestinal system complications and that cardiac involvement occurred at a similar rate $(6.1 \%)$, whereas respiratory involvement was slightly lower (6.8\%).

Although we have limited data on genetics, the results of our study might provide a general idea of the underlying genetic abnormalities in Turkish patients with aHUS. It has recently been reported that $60-70 \%$ of patients with aHUS carry identifiable mutations in complement genes or anti-CFH antibodies, which result in TMA lesions [11]. In a multicenter audit analysis comprising 71 patients, $61 \%$ of the patients with results available for both complement genetic testing and anti $\mathrm{CFH}$-autoantibody testing had evidence of alternative complement pathway dysregulation [12]. In our study population, genetic analyses were completed for the corresponding genes in 42 patients (37.1\%). In this subset 
of patients, disease-causing mutations, 17 of which were novel, were identified in 34 patients (80.9\%). This figure might indicate that the probability of detecting any mutation in already known genes is high in Turkish patients and, thereby, justifies the necessity of genetic screening for a long-term therapeutic plan. In previous studies, CFH mutations were the most commonly identified abnormalities [2, 18]. In contrast, we found that $M C P$ mutations and $C 3$ mutations were the most common genetic abnormalities whereas $C F H$ mutations were detected in less than $10 \%$ of the genetically studied population. These initial results may confirm the racial differences between Turks and other populations. One of the limitations of our study was that we did not screen for thrombomodulin mutations, which have a minor role in aHUS [11].

Before the introduction of eculizumab, plasma therapy was the mainstay of treatment for aHUS, based on the premise that it was beneficial by supplying normal complement proteins and/or removing mutant proteins or autoantibodies [11, 12]. After the study published by Bell et al. in 1991, plasma-based therapies became the initial treatment for most patients with aHUS [19]. Noris et al. reported that two-third of adult patients with aHUS had a poor outcome after 3 years of follow-up [3]. In a study by Johnson et al. on aHUS treatment outcomes in 71 patients, 59 patients received plasma therapy and the other 12 patients received other types of treatment. Their results revealed that $17 \%$ of the patients remained dependent on dialysis and $80.3 \%$ of the patients had persistent renal sequelae at day 33 of treatment. The longterm results of plasma therapy were unavailable [12]. In our study, $12.1 \%$ of the patients were on dialysis at the time of discharge; after a median follow-up period of 1.7 years, 1 patient was still on dialysis and 1 patient had undergone renal transplantation. These differences in findings between our study and other studies may be attributed to the different genetic backgrounds of the studied populations. Plasma therapy (i.e. plasma infusions and/or plasma exchange) still appears to be a valid therapeutic option for Turkish patients with aHUS.

The introduction of eculizumab has profoundly changed the management of aHUS. The efficacy of eculizumab has been demonstrated in three prospective clinical trials performed primarily in adult patients with TMA [14, 20, 21]. In these trials, it was demonstrated that eculizumab resulted in a time-dependent improvement in patients with aHUS, and the beneficial effects were maintained after 2 years of followup. In children, the evidence for the efficacy of eculizumab came from case reports and retrospective studies [11, 22, 23]. Recently, Greenbaum et al. published the first pediatric prospective study that assessed the efficacy and safety of eculizumab in patients with aHUS who were aged $<18$ years. Mutations in the genes of the alternative complement pathway were identified in $50 \%$ of the patients [15]. $M C P$ mutations were the most common; the mean duration of eculizumab treatment was 5.5 months, and hematological remission occurred in $82 \%$ of the patients. The authors reported that the mean improvement in eGFR from baseline to week 27 was $64 \mathrm{~mL} / \mathrm{min} / 1.73 \mathrm{~m}^{2}$, and discontinuation of dialysis was possible in $82 \%$ of the patients who initially required renal replacement therapy [15]. In our study, 16 patients (15.5\%) received eculizumab as a first-line therapy. Complete hematologic and renal remission was achieved in $82.2 \%$ and $76.3 \%$ of the patients, respectively. Our study as well as the study by Greenbaum et al. supported the efficacy of eculizumab in the acute period. However, there is ongoing debate on the appropriate duration and intervals for eculizumab treatment. Ardissino et al. reported findings from 10 patients who received eculizumab treatment, which was discontinued later. All of these patients had complement system dysregulation, and showed beneficial effects after being treated with eculizumab. After the eculizumab treatment was stopped, 3 patients relapsed within 6 weeks [24]. Later, the authors published results from a longer follow-up period. During a cumulative time off treatment of 243 months, 5 of the patients experienced relapse within 6 months of their last eculizumab dose [25]. In our patient cohort, eculizumab was discontinued in 52 patients for various reasons. The analyses of the genetic backgrounds and clinical outcomes of the patients are still underway and will be reported in detail in another manuscript.

Another noteworthy feature of our study is that we completed almost 2 years of patient follow-up. It has been previously reported that the mortality rate in children with aHUS was higher than that in adults, but the renal outcomes were worse in adults than in children [2]. In our study on pediatric patients, the mortality rate was $2 \%$ (3 out of 146 patients) during the acute stage, and $77.7 \%$ of the patients had a GFR $>90 \mathrm{~mL} / \mathrm{min} /$ $1.73 \mathrm{~m}^{2}$ during the follow-up period. Our results suggest that aHUS is a treatable disease and that progression to ESKD can be prevented with early diagnosis, and meticulous treatment and follow-up. However, it should be noted that in our patient cohort, high frequency of $M C P$ mutations, lack of information on completed genetic data and early initiation of treatment may explain favorable outcome.

\section{Conclusions}

We have established a unique Turkish aHUS registry that will increase our knowledge of patients with aHUS who have different genetic backgrounds, and enable the 
evaluation of different treatment options and patient outcomes. This registry is now open to adult patients. The collection of data on adult patients with aHUS will help researchers to better characterize the spectrum of adult aHUS in the country and will enable comparisons with pediatric cases.

\begin{abstract}
Abbreviations
aHUS: Atypical hemolytic uremic syndrome; C3: Complement factor 3; CFB: Complement factor B; CFH: Complement factor H; CFI: Complement factor I; CK: Creatine kinase; CFH-CFHR: Complement factor H-complement factor $\mathrm{H}$ related proteins; DGKE: Diacylglycerol kinase-E; eGFR: Estimated glomerular filtration rate; ESKD: End-stage kidney disease; GI: Gastrointestinal; IQR: Interquartile range; MCP (CD46): Membrane cofactor protein; MLPA: Multiplex ligation-dependent probe amplification; NRS: National registry system; SD: Standard deviation; STEC: Shiga toxin-producing E. coli; THBD: Thrombomodulin; TMA: Thrombotic microangiopathy
\end{abstract}

\section{Acknowledgements}

The authors acknowledge the enrolling clinicians, data coordinators at individual enrolling centers including Banu Çelikel Acar, Harika Alpay, Özlem Aydoğ, Esra Baskın, Aysun Karabay Bayazıt, Nur Canpolat, Nilgün Çakar, Ali Delibaş, Zelal Ekinci, Kibriya Fidan, Gürkan Genç, Nilufer Göknar, Can Hüzmeli, Beltinge Demircioğlu Kilıç, Mustafa Koyun, Engin Melek, Aytül Noyan, Elif Bahat Özdoğan, Sare Gülfem Özlü, Alper Soylu, Mehmet Taşdemir, Emel Ünsür, Lokman Üstyol, Bilal Yıldız, Alev Yılmaz, Selçuk Yüksel, Fatoş Yalçınkaya.

\section{Funding}

This study was supported by Hacettepe University Scientific Research and Development Office (Grant No: 010A101009). Article processing charge of this manuscript has been granted by the Alexion Pharmaceuticals, Inc.

\section{Availability of data and materials}

The datasets during and/or analysed during the current study available from the corresponding author on reasonable request.

\section{Authors' contributions}

NB conceived the study, and participated in its design. BG participated in the collection of data, contributed to the statistical analysis and drafted the manuscript. OS participated in designing of the study and in coordination of the clinical centers. ZBO participated in designing of the study and collection of data. EK carried out the molecular genetic studies. MH performed statistical analyses. FO conceived the study, participated in its design and coordination, evaluated genetic results, participated in analysis of data and wrote the manuscript. All authors have also contributed to manuscript development and review, and have read and approved the final manuscript.

\section{Competing interests}

Fatih Ozaltin and Oguz Soylemezoglu have received lecture and consultancy honoraria from the Alexion Pharmaceuticals, Inc.

\section{Ethics approval and consent to participate}

The study was approved by the ethics committee of Hacettepe University (FON10/03-22). Written informed consent was obtained from the parents of all the patients.

\section{Statement of prior presentation}

Aspects of these data were presented at the following congresses:

- Besbas N, Soylemezoglu O, Gulhan B, Ozcakar ZB, Korkmaz E, Ozaltin F. Turkish atypical haemolytic uremic syndrome registry: initial pediatric results. J Am Soc Nephrol 2015;26:457A. Presented at the American Society of Nephrology Kidney Week 2015, November 3-8, 2015, San Diego, CA, USA.

- N. Besbas, O. Soylemezoglu, B.Gulhan, ZB. Ozcakar, E. Korkmaz, M. Hayran, F. Ozaltin. Turkish Atypical Hemolytic Uremic Syndrome Registry: Evaluation of 146 Patients. Pediatr Nephrol 2016; 31:1884, PO-374. Presented at the 17th Congress of the International Pediatric Nephrology Association, September 20-24, 2016, Iguaçu, Brazil.

\section{Author details}

'Department of Pediatric Nephrology, Nephrogenetics Laboratory, Hacettepe University Faculty of Medicine, Sihhiye 06100, Ankara, Turkey. ${ }^{2}$ Department of Pediatric Nephrology, Gazi University Faculty of Medicine, Ankara, Turkey. ${ }^{3}$ Department of Pediatric Nephrology, Ankara University Faculty of Medicine, Ankara, Turkey. ${ }^{4}$ Department of Preventive Oncology, Hacettepe University, Ankara, Turkey.

Received: 21 September 2016 Accepted: 17 December 2016

Published online: 05 January 2017

\section{References}

1. Waters AM, Licht C. aHUS caused by complement dysregulation: new therapies on the horizon. Pediatr Nephrol. 2011;26:41-57.

2. Fremeaux-Bacchi V, Fakhouri F, Garnier A, Bienaimé F, Dragon-Durey MA, $\mathrm{Ngo}$ S, et al. Genetics and outcome of atypical hemolytic uremic syndrome: a nationwide French series comparing children and adults. Clin J Am Soc Nephrol. 2013;8:554-62.

3. Noris M, Caprioli J, Bresin E, Mossali C, Pianetti G, Gamba S, et al. Relative role of genetic complement abnormalities in sporadic and familial aHUS and their impact on clinical phenotype. Clin J Am Soc Nephrol. 2010;5:1844-59.

4. Geerdink LM, Westra D, van Wijk JA, Dorresteijn EM, Lilien MR, Davin JC, et al. Atypical hemolytic uremic syndrome in children: complement mutations and clinical characteristics. Pediatr Nephrol. 2012;27:1283-91.

5. Bernabéu-Herrero $M E$, Jiménez-Alcázar $M$, Anter J, Pinto $S$, Sánchez Chinchilla D, Garrido S, et al. Complement factor H, FHR-3 and FHR-1 variants associate in an extended haplotype conferring increased risk of atypical hemolytic uremic syndrome. Mol Immunol. 2015;67:276-86.

6. Lee JM, Park YS, Lee JH, Park SJ, Shin JI, Park YH, et al. Atypical hemolytic uremic syndrome: Korean pediatric series. Pediatr Int. 2015;57:431-8.

7. Sinha A, Gulati A, Saini S, Blanc C, Gupta A, Gurjar BS, et al. Prompt plasma exchanges and immunosuppressive treatment improves the outcomes of anti-factor $\mathrm{H}$ autoantibody-associated hemolytic uremic syndrome in children. Kidney Int. 2014;85:1151-60.

8. Lemaire M, Frémeaux-Bacchi V, Schaefer F, Choi M, Tang WH, Le Quintrec $\mathrm{M}$, et al. Recessive mutations in DGKE cause atypical hemolytic-uremic syndrome. Nat Genet. 2013;45:531-6.

9. Ozaltin F, Li B, Rauhauser A, An SW, Soylemezoglu O, Gonul I, et al. DGKE variants cause a glomerular microangiopathy that mimics membranoproliferative GN. J Am Soc Nephrol. 2013;24:377-84.

10. Verhave JC, Wetzels JF, van de Kar NC. Novel aspects of atypical haemolytic uraemic syndrome and the role of eculizumab. Nephrol Dial Transplant. 2014;29 Suppl 4:iv131-41.

11. Loirat C, Fakhouri F, Ariceta G, Besbas N, Bitzan M, Bjerre A, et al. An international consensus approach to the management of atypical haemolytic uremic syndrome in children. Pediatr Nephrol. 2016;31:15-39.

12. Johnson S, Stojanovic J, Ariceta G, Bitzan M, Besbas N, Frieling M, et al. An audit analysis of a guideline for the investigation and initial therapy of diarrhea negative (atypical) hemolytic uremic syndrome. Pediatr Nephrol. 2014;29:1967-78.

13. Delvaeye M, Noris M, De Vriese A, Esmon CT, Esmon NL, Ferrell G, et al. Thrombomodulin mutations in atypical hemolytic-uremic syndrome. N Engl Med. 2009;361:345-57.

14. Legendre CM, Licht C, Muus P, Greenbaum LA, Babu S, Bedrosian C, et al. Terminal complement inhibitor eculizumab in atypical hemolytic-uremic syndrome. N Engl J Med. 2013;368:2169-81.

15. Greenbaum LA, Fila M, Ardissino G, Al-Akash SI, Evans J, Henning P, et al. Eculizumab is a safe and effective treatment in pediatric patients with atypical hemolytic uremic syndrome. Kidney Int. 2016;89:701-11.

16. Licht C, Ardissino G, Ariceta G, Cohen D, Cole JA, Gasteyger C, et al. The global aHUS registry: methodology and initial patient characteristics. BMC Nephrol. 2015;16:207.

17. Maga TK, Nishimura CJ, Weaver AE, Frees KL, Smith RJ. Mutations in alternative pathway complement proteins in American patients with atypical hemolytic uremic syndrome. Hum Mutat. 2010;31:E1445-60.

18. Neumann HP, Salzmann M, Bohnert-Iwan B, Mannuelian T, Skerka C, Lenk D, et al. Haemolytic uraemic syndrome and mutations of the factor $\mathrm{H}$ gene: a registry-based study of German speaking countries. J Med Genet. 2003;40:676-81. 
19. Bell WR, Braine HG, Ness PM, Kickler TS. Improved survival in thrombotic thrombocytopenic purpura-hemolytic uremic syndrome. Clinical experience in 108 patients. N Engl J Med. 1991;325:398-403.

20. Licht C, Greenbaum LA, Muus P, Babu S, Bedrosian CL, Cohen DJ, et al. Efficacy and safety of eculizumab in atypical hemolytic uremic syndrome from 2-year extensions of phase 2 studies. Kidney Int. 2015;87:1061-73.

21. Fakhouri F, Hourmant M, Campistol JM, Cataland SR, Espinosa M, Gaber AO, et al. Terminal complement inhibitor eculizumab in adult patients with atypical hemolytic uremic syndrome: a single-Arm, Open-Label Trial. Am J Kidney Dis. 2016;68:84-93.

22. Besbas N, Gulhan B, Karpman D, Topaloglu R, Duzova A, Korkmaz E, et al. Neonatal onset atypical hemolytic uremic syndrome successfully treated with eculizumab. Pediatr Nephrol. 2013;28:155-8.

23. Baskin E, Gülleroğlu K, Kantar A, Bayrakci U, Ozkaya O. Success of eculizumab in the treatment of atypical hemolytic uremic syndrome. Pediatr Nephrol. 2015;30:783-9.

24. Ardissino G, Testa S, Possenti I, Tel F, Paglialonga F, Salardi S, et al. Discontinuation of eculizumab maintenance treatment for atypical hemolytic uremic syndrome: a report of 10 cases. Am J Kidney Dis. 2014;64:633-7

25. Ardissino G, Possenti I, Tel F, Testa S, Salardi S, Ladisa V. Discontinuation of eculizumab treatment in atypical hemolytic uremic syndrome: an update. Am J Kidney Dis. 2015;66:172-3.

26. Caprioli J, Castelletti F, Bucchioni S, Bettinaglio P, Bresin E, Pianetti G, et al, Complement factor $\mathrm{H}$ mutations and gene polymorphisms in haemolytic uraemic syndrome: the C-257 T, the A2089G and the G2881T polymorphisms are strongly associated with the disease. Hum Mol Genet. 2003;12:3385-95.

27. Richards A, Buddles MR, Donne RL, Kaplan BS, Kirk E, Venning MC, et al. Factor $\mathrm{H}$ mutations in hemolytic uremic syndrome cluster in exons $18-20$, a domain important for host cell recognition. Am J Hum Genet. 2001;68:485-90.

28. Kavanagh D, Yu Y, Schramm EC, Triebwasser M, Wagner EK, Raychaudhuri S, et al. Rare genetic variants in the CFI gene are associated with advanced age-related macular degeneration and commonly result in reduced serum factor I levels. Hum Mol Genet. 2015;24:3861-70.

29. Bresin E, Rurali E, Caprioli J, Sanchez-Corral P, Fremeaux-Bacchi V, Rodriguez De Cordoba S, et al. Combined complement gene mutations in atypical hemolytic uremic syndrome influence clinical phenotype. Am Soc Nephrol. 2013;24:475-86

30. Fremeaux-Bacchi V, Moulton EA, Kavanagh D, Dragon-Durey MA, Blouin J, Caudy A, et al. Genetic and functional analyses of membrane cofactor protein (CD46) mutations in atypical hemolytic uremic syndrome. J Am Soc Nephrol. 2006;17:2017-25.

31. Gilbert RD, Stanley LK, Fowler DJ, Angus EM, Hardy SA, Goodship TH. Cisplatininduced haemolytic uraemic syndrome associated with a novel intronic mutation of CD46 treated with eculizumab. Clin Kidney J. 2013;6:421-5.

32. Caprioli J, Bettinaglio P, Zipfel PF, Amadei B, Daina E, Gamba S, et al. The molecular basis of familial hemolytic uremic syndrome: mutation analysis of factor $\mathrm{H}$ gene reveals a hot spot in short consensus repeat 20. J Am Soc Nephrol. 2001;12:297-307.

33. Roumenina LT, Jablonski M, Hue C, Blouin J, Dimitrov JD, Dragon-Durey MA, et al. Hyperfunctional C3 convertase leads to complement deposition on endothelial cells and contributes to atypical hemolytic uremic syndrome. Blood. 2009;1 14:2837-45.

34. Feng S, Eyler SJ, Zhang Y, Maga T, Nester CM, Kroll MH, et al. Partial ADAMTS13 deficiency in atypical hemolytic uremic syndrome. Blood. 2013; 122:1487-93.

\section{Submit your next manuscript to BioMed Central and we will help you at every step:}

- We accept pre-submission inquiries

- Our selector tool helps you to find the most relevant journal

- We provide round the clock customer support

- Convenient online submission

- Thorough peer review

- Inclusion in PubMed and all major indexing services

- Maximum visibility for your research

Submit your manuscript at www.biomedcentral.com/submit

) Biomed Central 